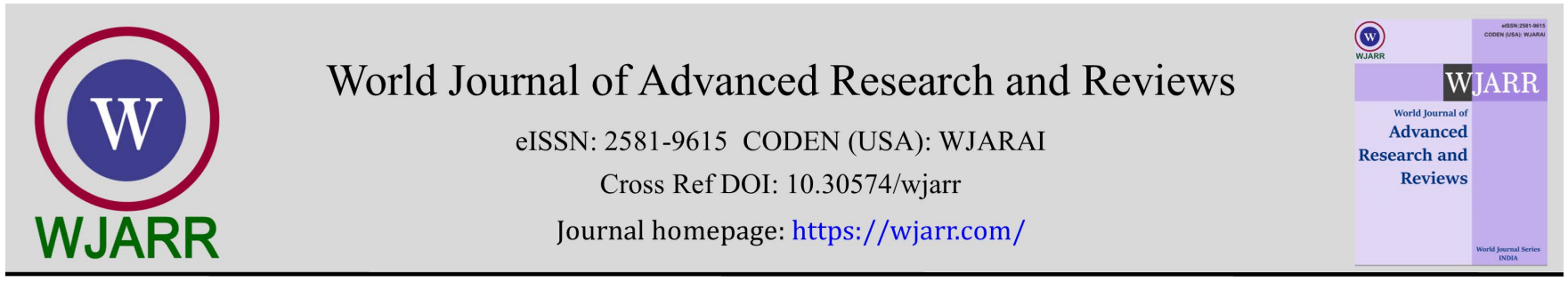

(CASE REPORT)

Check for updates

\title{
Rickettsioses and Covid-19: About one case
}

Ilyass Laaribi 1,2, *, Hamza Mimouni 1,2, Ghizlane El aidouni 1,2, Abdelilah El Rhalete 1, 2, Houssam Bkiyar 1, 2, 3 and Brahim Housni 1,2,3

${ }^{1}$ Department of Intensive Care Unit, Mohammed VI University Hospital, Oujda, Morocco.

2 Faculty of Medicine and Pharmacy, Mohammed First University, Oujda, Morocco.

${ }^{3}$ Mohammed First University Oujda, FMP Oujda, LAMCESM, Oujda, Morocco.

World Journal of Advanced Research and Reviews, 2022, 13(02), 501-504

Publication history: Received on 11 January 2022; revised on 12 February 2022; accepted on 14 February 2022

Article DOI: https://doi.org/10.30574/wjarr.2022.13.2.0157

\begin{abstract}
Introduction: Covid-19 infection is an emerging infectious disease whose clinical features have been widely described over the past 2 years, cutaneous involvement remains relatively uncommon and may be confused with other skin manifestations.

Case presentation and discussion: We report the case of a patient who presented a clinical picture associating a Covid19 infection and Rickettsiosis, we will discuss these pathologies while emphasizing the importance of thinking about differential diagnoses in front of a Covid-19 infection.
\end{abstract}

Conclusion: In this pandemic context, clinicians must remain vigilant regarding other febrile rashes that can be mistaken for a Covid-19 infection and leading to delays in diagnosis and treatment.

Keywords: Covid-19; SARS-CoV-2; Dermatological manifestations; Rickettsiosis

\section{Introduction}

Covid-19 infection is an emerging infectious disease whose clinical features have been widely described over the past 2 years, with mainly fever and respiratory symptoms [1], but over time several other cardiovascular, neurological and digestive manifestations have been reported [2]. Dermatological manifestations remain considered infrequent presentations of Covid-19 [3]. Some of these skin lesions can be confused with other cutaneous manifestations observed in certain infections such as Rickettsiosis [4].

We report the case of a 54-year-old patient hospitalized in intensive care for a Covid-19 infection associated with late diagnosed Rickettsiosis.

\section{Case presentation}

54-year-old patient, with no previous medical history, not vaccinated against Covid-19, who consults in the emergency department for asthenia, fever, severe headaches, myalgia, diarrhea, skin rash and respiratory distress evolving for 5 days. On interrogation, his family reports the notion of an influenza-like syndrome 7 days preceding the current symptomatology.

\footnotetext{
${ }^{*}$ Corresponding author: Ilyass laaribi

Department of Intensive Care Unit, Mohammed VI University Hospital, Oujda,Morocco.

Copyright $(2022$ Author(s) retain the copyright of this article. This article is published under the terms of the Creative Commons Attribution Liscense 4.0.
} 
The clinical examination found a confused patient Glascow Coma Score $13 / 15$, febrile at $41^{\circ} \mathrm{C}$, without neck stiffness, tachycardic with regular heart rate of 126 beats per minute, blood pressure at $138 / 72 \mathrm{~mm}^{3} \mathrm{Hg}$, a respiratory rate of 38 breaths per minute, arterial oxygen saturation of $78 \%$ in ambient air and $93 \%$ on 41 / min of oxygen. Skin examination finds a generalized erythematous maculopapular rash eruption involving palms, with no inoculation eschar (Figure 1).

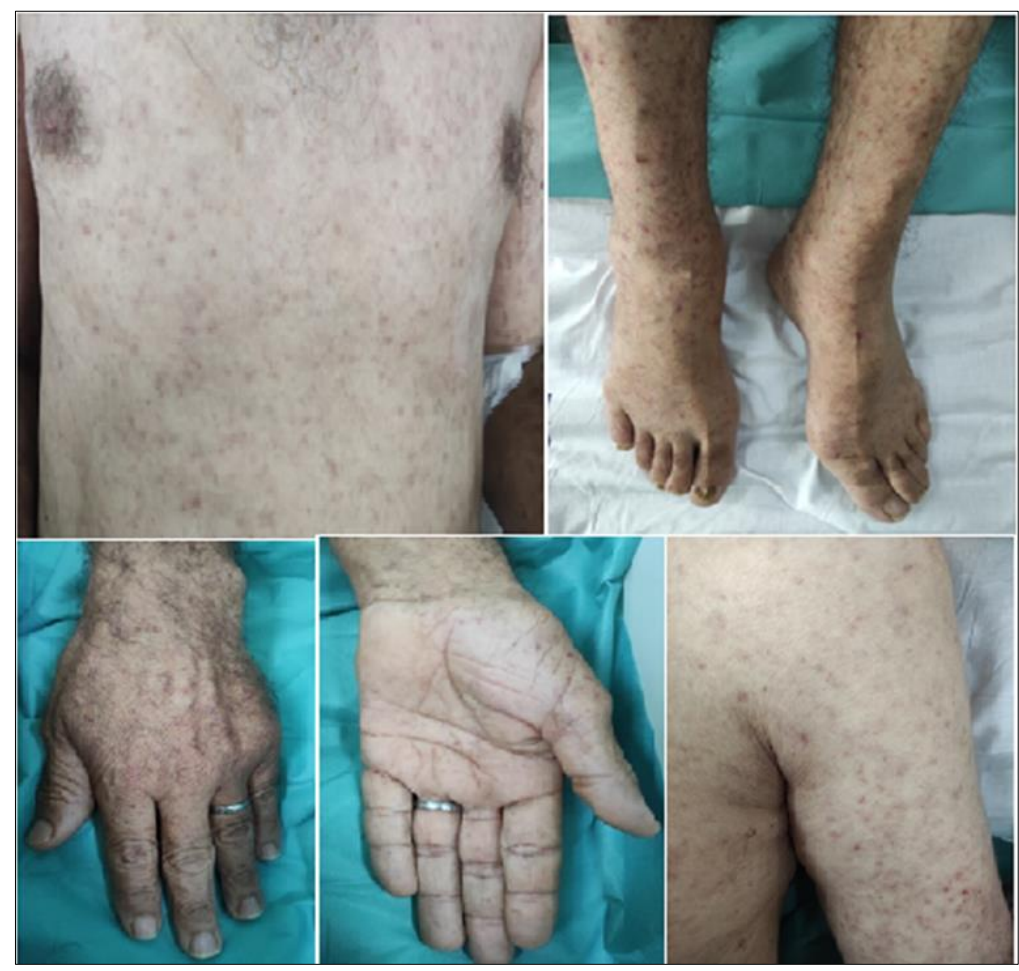

Figure 1 Maculopapular rash involving the whole body

The patient's full blood count revealed a hyperleukocytosis at $12,800 / \mathrm{mm}^{3}$, lymphopenia at $780 / \mathrm{mm}^{3}$, a platelet count at $62,000 / \mathrm{mm}^{3}$, a very perturbed inflammatory assessment with a raised C-reactive protein ( $\left.247 \mathrm{mg} / \mathrm{ml}\right)$, ferritinemia at $2240 \mathrm{mcg}$ / L, procalcitonin at $6.49 \mathrm{ng} / \mathrm{ml}$, IL6 $98 \mathrm{pg} / \mathrm{ml}, \mathrm{LDH} 874 \mathrm{IU}$ / l, hepaticcytolysis, and a positive Sars Cov-2 PCR.

To explore his confusion, a brain CT scan (Figure 2) followed by a lumbar puncture was performed returning normal. The patient underwent thoracic CT which found an involvement compatible with a Covid-19 infection estimated between $25 \%$ and $50 \%$ (Figure 3).
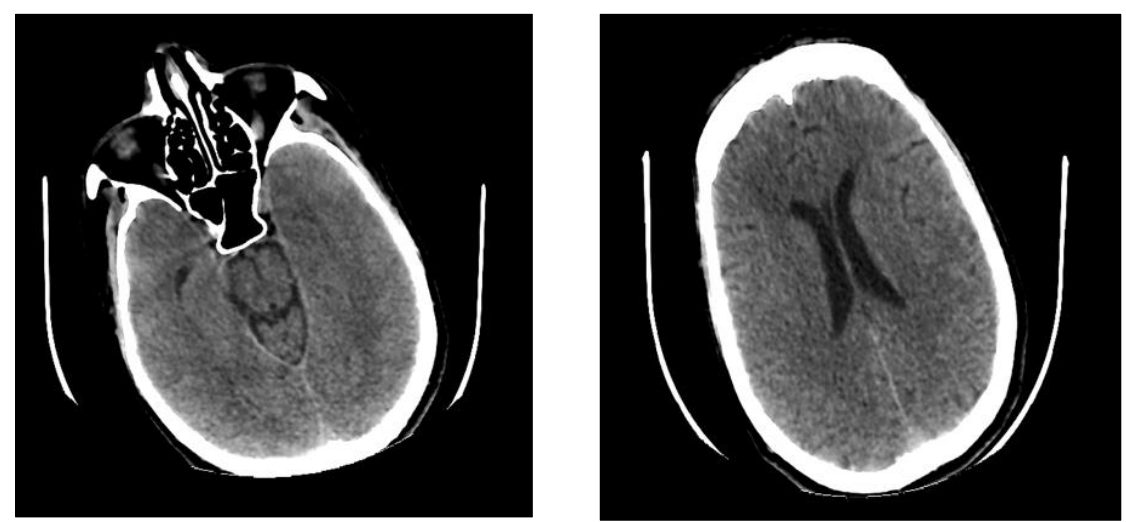

Figure 2 Normal CT brain scan 


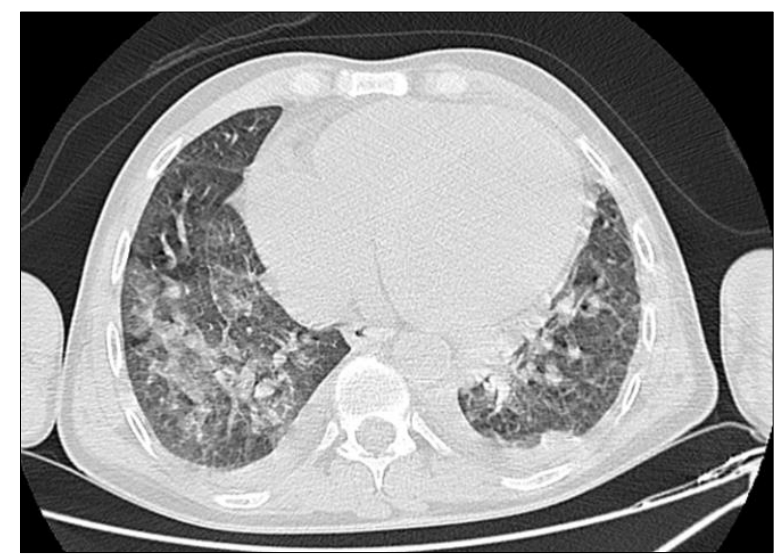

Figure 3 Axial sequence of a lung window chest-CT showing typical Covid-19 lesions with an estimated reach between 25 and $50 \%$

The patient was put on Ceftriaxone $2 \mathrm{~g} / \mathrm{d}$, Dexamethasone $6 \mathrm{mg} / \mathrm{d}$, Vitamin C and Zinc.

Giventhe persistence of fever 5 days after admission and the appearance of skin lesions, Rickettsiosis was suspected and antibiotic therapy with Doxycycline $100 \mathrm{mg}$ twice daily probabilistically was added.

After 3 days, the gradual improvement in the state of consciousness began to be noted, the regression of fever and skin lesions, the re-ascent of platelets and the normalization of the liver function; the patient was weaned from oxygen and he returned home after three weeks of hospitalization. A serology was requested on his discharge from the hospital which came back positive confirming the diagnosis of Rickettsiosis.

\section{Discussion}

Some patients with Covid-19 may present with dermatological manifestations [5], in addition to these skin lesions, patients may present with fever which is a widely recognized symptom of Covid-19. Therefore, other febrile illnesses can be difficult to distinguish leading to diagnostic and therapeutic delays, especially in this pandemic context [6].

Skin lesions from Covid-19 may resemble those commonly seen in Rickettsioses, for example, both diseases can cause maculopapular, vesicular, vascular lesions and rashes. Covid-19 and Rickettsioses can be distinguished by the presentation most often of an inoculation eschar, however, this eschar can be absent at times which was the case in our patient [7].

Rickettsiosis is a zoonosis with worldwide distribution caused by intracellular bacteria that preferentially infect endothelial cells, it is transmitted to humans by lice, fleas, ticks and mites. The classic clinical presentation of Rickettsiosisis a triad of sudden onset fever, headache and rash, occurring two to fourteen days after a bite from an infected arthropod [8].

This symptomatology can be neglected due to the pandemic context and the overlap with the symptoms of Covid-19, other symptoms have been reported including neurological symptoms (confusion, convulsions in 15 to $45 \%$ ), cough $35 \%$ and gastrointestinal symptoms 26 to $40 \%$, in the laboratory assessment we can find a disturbance of the hepatic assessment, an elevation of the lactate dehydrogenase, a thrombocytopenia and hyponatremia [9].

The diagnosis is initially based on epidemiological, clinical (fever, headache, rash) and paraclinical (thrombocytopenia and hepatic cytolysis) criteria. In clinical practice serology is the test of reference in most laboratories which only allows retrospective diagnosis.[10], Nucleic acid amplification (PCR) and immunohistochemistry allow a diagnosis in the acute stage of infection, but are not available in our context.

As soon as the diagnosis of Rickettsiosis is suspected, empirical treatment should be started even before the diagnosis is confirmed in order to avoid potentially fatal complications. Doxycycline is the treatment of choice [11]. In our case giventhe persistence of fever in the patient and the appearance of skin lesions even with a Covid-19 infection confirmed by PCR, treatment with Doxycycline was started with spectacular improvement that is why it is important for all clinicians to think about other conditions that can cause persistent fever. 


\section{Conclusion}

The dermatological manifestations of Covid-19 and Rickettsioses have several similarities, however, patients with Rickettsioses can be easily treated with Tetracyclines, while there is not yet a clear consensus on the treatment of patients with Covid -19 , for this reason during this pandemic, clinicians must be vigilant to other febrile illnesses whose symptoms overlap with a Covid-19 infection in order to avoid any delay in treatment.

\section{Compliance with ethical standards}

\section{Acknowledgments}

This research did not receive any funding.

\section{Disclosure of conflict of interest}

The authors declare no conflict of interest.

\section{Statement of informed consent}

Informed consent was obtained from all individual participants included in the study.

\section{References}

[1] Huang C, Wang Y, Li X, Ren L, et al. Clinical features of patients infected with 2019 novel coronavirus in Wuhan, China. Lancet. 15 Feb 2020;395(10223):497-506.

[2] Gupta A, Madhavan MV, Sehgal K, Nair N, Mahajan S, Sehrawat TS, et al . Extrapulmonary manifestations of COVID19. Nat Med. Jul 2020;26(7):1017-1032.

[3] Suchonwanit P, Leerunyakul K, Kositkuljorn C. Cutaneous manifestations in COVID-19: Lessons learned from current evidence. J Am AcadDermatol. Jul 2020;83(1):e57-e60.

[4] Almutairi N, Schwartz RA. COVID-19 with dermatologic manifestations and implications: An unfolding conundrum. Dermatol Ther. Sep 2020;33(5):e13544.

[5] Daneshgaran G, Dubin DP, Gould DJ. Cutaneous Manifestations of COVID-19: An Evidence-Based Review. Am J Clin Dermatol. Oct 2020;21(5):627-639.

[6] Coleman JJ, Manavi K, Marson EJ, Botkai AH, Sapey E. COVID-19: to be or not to be; that is the diagnostic question. Postgrad Med J. Jul 2020; 96(1137): 392-398.

[7] Mittal A, Elias ML, Schwartz RA, Kapila R. Recognition and treatment of devastating vasculopathic systemic disorders: Coronavirus disease 2019 and rickettsioses. Dermatol Ther. Jul 2021;34(4):e14984.

[8] Portillo A, Santibáñez S, García-Álvarez L, Palomar AM, Oteo JA. Rickettsioses in Europe. Microbes Infect. NovDec 2015;17(11-12): 834-8.

[9] Boillat N, Greub G. Approcheclinique des rickettsioses, Rev Med Suisse. 2007; 7(111): 1222 - 1227.

[10] Fournier PE, Jensenius M, Laferl H, Vene S, Raoult D. Kinetics of antibody responses in Rickettsia africae and Rickettsia conorii infections. Clin Diagn Lab Immunol. 2002;9(2):324-328.

[11] Blanton LS. The Rickettsioses: A Practical Update. Infect Dis Clin North Am. 2019 Mar;33(1):213-229. 